\title{
Development of a Gas-Fed Plasma Source for Pulsed High-Density Plasma/Material Interaction Studies
}

\author{
Michael V. Pachuilo, Francis Stefani, Laxminarayan L. Raja, Roger D. Bengtson, Graeme A. Henkelman, \\ A. Cuneyt Tas, Waltraud M. Kriven, and Kumar Sinha Suraj
}

\begin{abstract}
A gas-fed capillary plasma source has been developed to study plasma-surface interactions under pulsed high pressure arc conditions, without the use of an exploding fuse wire or ablative liner. A nonintrusive preionization source has been developed to break down relatively large interelectrode gaps at low charge voltages of 2-6 kV. The preionization source comprises a nonequilibrium surface streamer discharge that forms a conducting channel through which the main thermal arc discharge is initiated. The arc electron temperature and number density are estimated to be $T_{e} \sim 1-2 \mathrm{eV}$ and $n_{e} \sim 10^{23} \mathrm{~m}^{-3}$. Silicon and sapphire samples were exposed to the arc plasma and revealed deposition of electrode and wall materials. Substitution of Elkonite $50 \mathrm{~W} 3$ for brass electrodes reduced plasma contamination to acceptable levels. The plasma-material interactions were examined and quantified using scanning electron microscopy and energy dispersive $\mathrm{X}$-ray spectroscopy.
\end{abstract}

Index Terms-Arc discharge, atmospheric discharge, capillary discharge, plasma-material interaction, pulsed thermal plasma.

\section{BACKGROUND}

$\mathbf{H}$ IGH-POWER, high-pressure, capillary-plasma discharges have a variety of applications in pulsed space propulsion devices and as igniters for chemical propellant guns [1]-[5]. The plasma formed in these devices is sustained through the ablation of a sacrificial liner material (typically a polymer).

Manuscript received October 31, 2013; revised January 13, 2014, February 18, 2014, May 14, 2014, and June 24, 2014; accepted July 18, 2014. Date of publication August 13, 2014; date of current version October 21, 2014. This work was supported by the Air Force Office of Scientific Research, Arlington, VA, USA, under Contract FA9550-11-1-0062.

M. V. Pachuilo is with the Department of Aerospace Engineering and Engineering Mechanics, University of Texas at Austin, Austin, TX 78712 USA (e-mail: mvpachuilo@utexas.edu).

F. Stefani is with the Center for Aeromechanics Research, University of Texas at Austin, Austin, TX 78712 USA (e-mail: stefani@utexas.edu).

L. L. Raja is with the Department of Aerospace Engineering and Engineering Mechanics, University of Texas at Austin, Austin, TX 78712 USA, and also with the Center for Aeromechanics Research, University of Texas at Austin, Austin, TX 78712 USA (e-mail: 1raja@mail.utexas.edu).

R. D. Bengtson is with the Department of Physics, University of Texas at Austin, Austin, TX 78712 USA (e-mail: bengtson@physics.utexas.edu).

G. A. Henkelman is with the Department of Chemistry, University of Texas at Austin, Austin, TX 78712 USA (e-mail: henkelman@cm.utexas.edu).

A. C. Tas and W. M. Kriven are with the Department of Materials Science and Engineering, University of Illinois at Urbana-Champaign, Champaign, IL 61801 USA (e-mail: actas@illinois.edu; kriven@illinois.edu).

K. S. Suraj is with the Department of Physics, Pondicherry University, Puducherry 605014, India (e-mail: suraj.kumar.sinha.pu@gmail.com).

Color versions of one or more of the figures in this paper are available online at http://ieeexplore.ieee.org.

Digital Object Identifier 10.1109/TPS.2014.2344974
Capillary plasmas are thermal arcs characterized by very high in-bore pressures $(\sim 1-10 \mathrm{MPa})$ with very high input power densities delivered to the plasma during a short pulse transient.

The high pressures, and correspondingly high collisionality, result in local thermodynamic equilibrium (LTE) conditions within the core of the arc. Typical LTE arc core temperatures are about 1-2 eV and charge particle densities of $\sim 10^{23}-10^{28} \mathrm{~m}^{-3}$ [6]. The arc column is typically spatially nonuniform and strong gradients in the arc properties exist. For example, the periphery of the arc column in the vicinity of the ablating surface has a much lower temperature of $\sim 0.1 \mathrm{eV}[7]$.

Material surfaces exposed to thermal arc plasmas encounter extreme conditions. The charged particle flux to a surface under these conditions is estimated at $\sim 10^{27} \mathrm{~m}^{-2} \mathrm{~s}^{-1}$ within the core of the arc [6]. Surface heat flux for the same conditions is $\sim 1 \mathrm{GW} / \mathrm{m}^{2}$ and can, therefore, cause substantial damage to plasma facing material components with repeated use [8].

An understanding of how thermal plasmas interact with material surfaces is key to the development of reliable, longlifetime capillary arc based devices. However, plasma-material interaction studies in these discharges are extremely difficult due to the small capillary bore size (approximately few millimeters in diameter). Such small diameters preclude direct placement of a material sample within the chamber bore.

The easiest approach to studying how these plasmas interact with a material surface is by expanding the plasma from the bore into an external plasma jet, and allowing it to interact with a sample surface placed in its path. The expansion process, however, results in a drop in pressure and temperature, and the plasma composition can be vastly different from the conditions within the capillary bore. For example, expanding the plasma from a bore at a pressure of $\sim 10 \mathrm{MPa}$ to ambient atmospheric conditions can result in a significant temperature drop from 1 to $\sim 0.1 \mathrm{eV}$ [9], [10]. At this temperature, several chemical components of the plasma (originating from the ablating liner) condense out and are deposited on the target material surface. Furthermore, practical arc breakdown (ignition) schemes in capillary discharges typically involve a metal fuse wire connecting the electrodes that is exploded to produce an initial gaseous plasma conducting channel. The metal vapor contamination from the fuse wire is also a major problem for plasma-material interaction studies. 


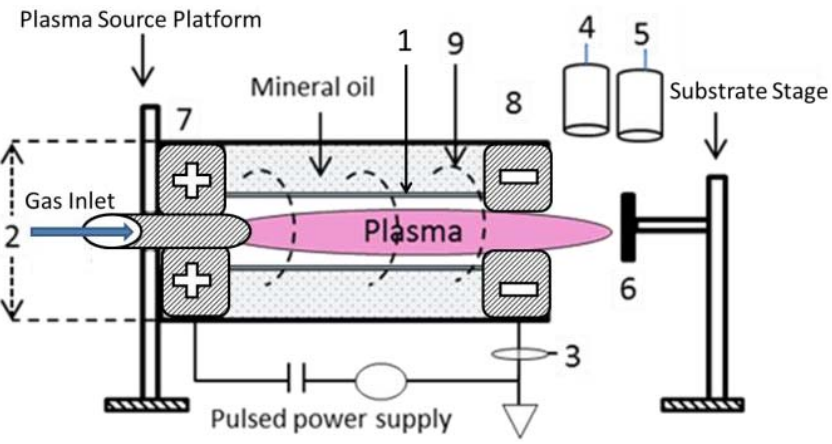

Fig. 1. Schematic of the experimental setup. The plasma discharge chamber is made up of two concentric tubes (1) the inner, fused quartz tube and (2) outer polycarbonate tube. These tubes are held in place with two annular electrodes at the ends (7) anode and (8) cathode. A copper ignition wire (9) is wound around the inner fused quartz tube, where high voltage is applied to trigger the discharge. The volume between the two tubes is filled with mineral oil. (6) Sample can be moved to different locations. (3)-(5) Represent a Rogowski coil, an optical emission spectroscopy, and a photodiode, respectively. The plasma jet exhausts to ambient atmospheric conditions.

This paper discusses a new gas-fed capillary source that alleviates several of the above problems with ablation-fed capillary sources, while providing a highly flexible and reliable pulsed thermal plasma source for plasma/material interaction studies. We developed a high-voltage, nonintrusive trigger approach to enable gas breakdown in high pressure, large interelectrode gaps, thereby eliminating the need for an exploding fuse wire. The new source also provides significant flexibility in the plasma composition through the choice of different feed gases, which in turn allows the possibility for a systematic study of chemical aspects of plasma/material interactions.

\section{EXPERIMENTAL SETUP}

\section{A. Experimental Overview}

A schematic of the experiment is shown in Fig. 1. The plasma reaction chamber has two annular electrodes located at the ends of the chamber with a trigger wire wrapped around the outer diameter of the fused quartz tube. Electrolytic capacitors, with a maximum voltage of $6 \mathrm{kV}$, provide power for the thermal arc discharge. Breakdown across the charged electrodes is initiated using a separate high-voltage pulse that is applied to the trigger wire. The high-voltage pulse initiates a nonequilibrium, surface streamer discharge that tracks along the inner surface of the quartz tube. When the discharge has sufficient ionization to form a conduction channel between the electrodes of the main capacitor bank, a steep rise in current across the main electrodes ensues as the stored electrical energy is transferred into the gas discharge forming a thermal plasma.

The plasma chemical composition is controlled through use of high purity gas cylinders. The feed gases are regulated, metered, and mixed before flowing into the reaction chamber. Background feed gases, such as argon, are metered through a 0-50 SLPM digital flow meter (Omega FMA-1609A). Small amounts of additive gases, such as hydrogen, oxygen, or methane, are metered through a $0-1$ SLPM meter (Omega FMA-1620A). Premixing of the background and additive gases occurs in a two meter long gas feed tube upstream

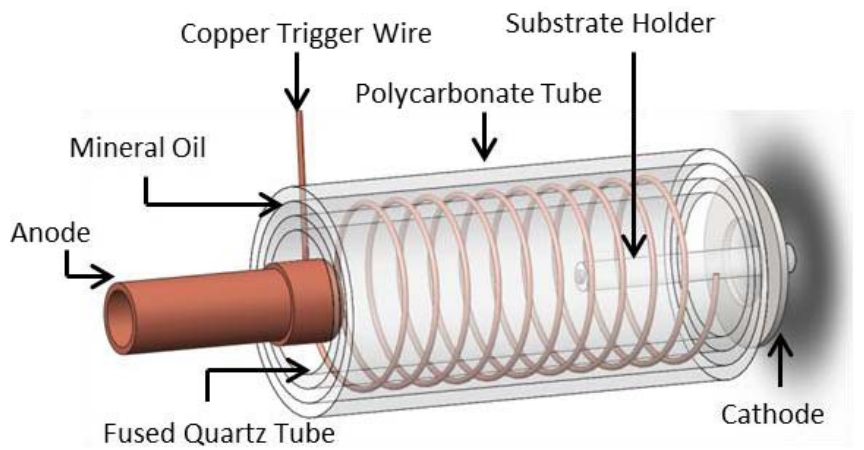

Fig. 2. Schematic of the plasma discharge chamber that consists of two concentric tubes, two annular end electrodes, and a helical copper wire used to initiate breakdown between the two electrodes.

of the chamber inlet. To ensure a uniform environment, the reaction chamber is purged for $5 \mathrm{~s}$ or more before initiating a discharge. Single component gases or gas mixtures are used to systematically investigate various plasma-surface reaction mechanisms. For example, a single species plasma composed of an inert noble gas is used to determine the importance of sputter mechanisms. Small amounts of oxidizing and reducing gases may be added to the inert background to investigate a chemically reactive plasma interacting with various materials. Materials exposed to the plasma include single crystal silicon, sapphire, and tungsten. Each of these materials is selected based upon their bond nature: covalent, ionic, or metallic, respectively.

The test samples are fixed to a substrate holder and inserted through the cathode into the discharge chamber. Samples are exposed to several pulsed, thermal plasma discharges, before surface analysis. The effects of various plasma environments on each benchmark material are experimentally investigated with various surface analysis tools, such as scanning electron microscopy (SEM) and energy dispersive X-ray spectroscopy (EDXS). The work presented in this paper focuses on the development and characterization of the plasma source. Details of the various experimental subsystems and diagnostics are presented in the remainder of this section.

\section{B. Plasma Discharge Chamber and Feed Gas System}

Fig. 2 shows a schematic of the plasma discharge chamber made up of two concentric tubes: an inner fused quartz tube, (100-mm long with a 32-mm inner diameter) and an outer polycarbonate tube. A 0.2 -mm-thick copper trigger wire is wound into a helix on the outside of the quartz tube. A negative polarity pulse $(-50 \mathrm{kV})$ is applied to the helical copper wire to trigger the gas breakdown, while the two annular discharge electrodes are held at a fixed potential. The cathode lies flush with the exit plane of the chamber, while the anode protrudes $10 \mathrm{~mm}$ into the plasma discharge chamber. The region between the fused quartz and polycarbonate tube is filled with mineral oil to insulate the system and avoid external breakdown during the $-50 \mathrm{kV}$ trigger pulse. The assembly is sealed at the ends by two Viton O-rings and a small amount of epoxy. The figure shows the anode (left), cathode (right), and an alumina 
substrate holder inserted through the cathode to which samples are affixed.

\section{Power Supply}

1) Pulsed Thermal Arc Discharge Supply: The pulsed power supply for the arc discharge is provided by $185 \mu \mathrm{F}$ (nominal) electrolytic capacitors with a peak charge voltage of $3 \mathrm{kV}$ (General Atomics part \# 39504). A direct-current Spellman SL600 power supply is used to charge the capacitors. The charge circuit is isolated from the pulsed discharge circuit before ignition through high-voltage Ross relay switches. The capacitors are arranged in various series-parallel combinations for different operating voltages and energies. The system is operated between 2 and $5 \mathrm{kV}$, therefore two capacitors must be connected in series. Additional capacitors are added in parallel pairs to vary the stored energy. The system has been operated up to a maximum stored energy of $4.45 \mathrm{~kJ}$ with eight capacitors in series-parallel configuration at a charge voltage of $5 \mathrm{kV}$. To minimize circuit ringing, a low inductance strip line connects the capacitor bank to the plasma discharge chamber. With four capacitors in a series-parallel configuration, the inductance of the circuit is about $500 \mathrm{nH}$, resulting in a slightly underdamped pulse.

2) Nonthermal Preionization Trigger Supply: A highvoltage trigger approach was used to generate a streamer discharge that initiates breakdown of the arc plasma, as described in [11]. The high voltage pulse is generated using a solid-state capacitive discharge ignition (CDI) module (Mallory Hyfire 6A) that generates a $500 \mathrm{~V}$ pulse, which is then stepped up and inverted through a Mallory automotive transformer to the $-50 \mathrm{kV}$ pulse. We modified the CDI module to be triggered directly from a BNC model 555 pulse delay generator, which controls the sequencing of the diagnostics relative to the ignition of the plasma discharge. The output of the transformer secondary is connected to the helical trigger wire.

\section{Instrumentation}

Characteristics of the thermal arc discharge are determined from voltage and current traces, spectrum analysis, time resolved light intensity, and high-speed photography. Voltage and current traces, as well as high-speed photography are used to characterize the preionization source. The thermal discharge current and voltage are measured using passive Rogowski coils and a Tektronics P5210 differential highvoltage probe. Voltage and current of the high-voltage trigger wire are measured using a Tektronics P6015A high-voltage probe and a Pearson 2877 current monitor. The transient stagnation gauge pressure is measured using a piezoelectric sensor (PCB Piezotronics 113B21). The pressure gauge is epoxied into a 7-mm outer diameter, 8-cm long fused quartz tube to minimize charge accumulation and interference. The time-integrated emission spectrum of the discharge is recorded with an Ocean Optics HR2000+ spectrometer. Time resolved intensity $(350-1100 \mathrm{~nm})$ of the discharge is recorded by a ThorLabs PDA36A photodiode and a Dalsa charge-coupled device framing camera that provides a minimum exposure of $1 \mu \mathrm{s}$.
Fig. 3. Trigger voltage and plasma Rogowski signal $d I / d t$ versus time. The large dip in the trigger voltage, or unloading, at $27 \mu$ s correlates with the formation of a strong streamer current and transition to an arc discharge. Small amounts of unloading at 6 , and $22 \mu \mathrm{s}$, produce weak streamers that are precursors to breakdown.

\section{Plasma SOURCE}

\section{A. Nonthermal Preionization Source}

A key feature of the device is the ability to reliably form thermal arcs at voltages significantly lower than those required for Paschen breakdown, without ablation feed mechanisms or a fuse wire ignition system. The system operates at atmospheric pressure with an interelectrode distance of $10 \mathrm{~cm}$. The pressure-distance $(p d)$ product of 7600 torr $\cdot \mathrm{cm}$ for the system would require approximately $100 \mathrm{kV}$ for breakdown in an argon background. The nonequilibrium streamer discharge produced by the high-voltage trigger pulse serves to circumvent the Paschen breakdown mechanism to realize significantly lower charge voltages of $2-5 \mathrm{kV}$ for the arc breakdown.

On application of the $-50 \mathrm{kV}$ pulse, the inner surface of the quartz tube polarizes. The applied voltage on the quartz dielectric surface provides a sufficient electric field for streamer formation and propagation. Streamers propagate from the discharge electrodes and travel along the quartz surface to bridge the interelectrode gap forming a conduction channel. Once the streamer channel has sufficient conductivity, the discharge capacitors become shorted by the channel, and the capacitor bank discharges.

Fig. 3 shows the trigger voltage pulse and Rogowski coil signal, $d I / d t$, as functions of time. The Rogowski signal measures the rate of change of current flow through the discharge circuit, including the plasma source. Change in the monitored Rogowski signal indicates the formation of a thermal discharge. A large step-like discontinuity in the trigger voltage at $27 \mu$ s indicates the formation of a streamer conduction channel. Before the unloading event at $27 \mu \mathrm{s}$, no current has flowed in the discharge circuit, though small unloading dips in the trigger voltage are realized at 6 and $22 \mu \mathrm{s}$. These small dips in voltage correspond to streamer events that do not have sufficient conductivity to allow the capacitor bank to discharge. At $27 \mu \mathrm{s}$, the Rogowski signal indicates that the conduction channel bridges the interelectrode gap with sufficient conductivity to discharge the capacitor bank. As the capacitors discharge, rapid Joule heating occurs 


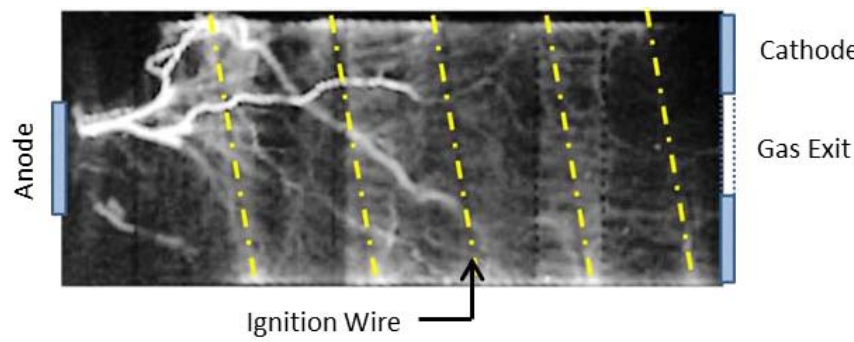

Fig. 4. High-speed image of streamers formed inside the plasma chamber In this photograph, the gas inlet and anode are on the left; the cathode is on the right. The feed gas is argon.

TABLE I

IgNition Threshold AND ARC DELAY DEPENDENCE ON CHARgE VOLTAGE FOR ARGON BACKGROUND

\begin{tabular}{|c|c|c|}
\hline $\begin{array}{c}\text { Charge } \\
\text { Voltage }\end{array}$ & $\begin{array}{c}\text { Ignition Threshold } \\
\text { Voltage }\end{array}$ & $\begin{array}{c}\text { Transition to Arc } \\
\text { Delay }\end{array}$ \\
\hline $1.6 \mathrm{kV}$ & - & $45 \mu \mathrm{s}$ \\
\hline $3.0 \mathrm{kV}$ & $40 \mathrm{kV}$ & $26 \mu \mathrm{s}$ \\
\hline $5.0 \mathrm{kV}$ & $5 \mathrm{kV}$ & $7 \mu \mathrm{s}$ \\
\hline
\end{tabular}

that leads to gas expansion and formation of an audible shockwave. The large current densities and high pressures result in a highly collisional plasma that is in thermodynamic equilibrium.

Fig. 4 shows an array of streamers inside the plasma chamber formed by the high-voltage trigger pulse. The streamers are highly branched, following a contorted path as they propagate in the discharge volume. The streamer array generates a volumetric source of seed electrons that provides sufficient ionization for thermal arc breakdown. The brightest streamer emission is observed at the left electrode, regardless of polarity. Enhanced light emission near the left electrode is due to asymmetry in the geometry of the electrode configuration. The left electrode protrudes $10 \mathrm{~mm}$ into the reaction chamber decreasing the breakdown threshold potential, whereas the right electrode is flush with the chamber exit.

The ignition threshold potential for a streamer that leads to the formation of a thermal arc depends on the applied potential across the main discharge electrodes. As the main interelectrode charge potential increases for an argon background, the trigger ignition threshold voltage for thermal arc formation decreases. Ignition threshold voltages may be defined as the voltage of the trigger pulse at which sufficient preionization has occurred to induce a thermal arc breakdown. The applied interelectrode potential reduces the required ignition voltage, and therefore, decreases the delay time for thermal arc formation for an argon background. Table I lists the ignition threshold voltages and transition to arc delay times as a function of charge voltage in an argon background. A charge voltage of 1.6 $\mathrm{kV}$ is the lowest potential for which breakdown was recorded. The dash indicates thermal breakdown is not reliable and the ignition threshold voltage occurred after the peak of trigger pulse.
TABLE II

RANGE OF OPERATING CONDITIONS WITH 99.5\% Pure ARgon FeEd GaS

\begin{tabular}{|l|c|c|c|}
\hline Operating conditions & Low & Typical & High \\
\hline Number of capacitors & 2 & 4 & 8 \\
\hline Capacitance, $\mu \mathrm{F}$ & 89 & 178 & 356 \\
\hline Charge voltage, $\mathrm{kV}$ & 1.6 & 3.2 & 5.0 \\
\hline Peak current, $\mathrm{kA}$ & 6 & 25 & 76 \\
\hline Plasma energy, $\mathrm{J}$ & 85 & 670 & 3100 \\
\hline Peak power, $\mathrm{MW}$ & 5 & 40 & 150 \\
\hline Pulse Duration, $\mu \mathrm{s}$ & 40 & 70 & 140 \\
\hline Peak pressure, $\mathrm{kPa}$ & $200 \pm 50$ & $1500 \pm 500$ & $>4000^{*}$ \\
\hline
\end{tabular}

* Pressure exceeds limit of pressure transducer

Fig. 5. Voltage, current, and light emission of the plasma at typical conditions.

\section{B. Thermal Plasma Arc Discharge}

The pulsed thermal plasma discharge source operates over a range of conditions. The high pressure, and therefore the collisionality, regulates the plasma temperature to approximately $1-2 \mathrm{eV}$. The plasma temperature is relatively insensitive to the stored capacitive energy, therefore various capacitor configurations will alter the $R C$ time constant and fluidic response even though plasma properties remain uniform.

The discharge has been operated with plasma durations of $40-140 \mu \mathrm{s}$, and with energies from $85 \mathrm{~J}$ to $3 \mathrm{~kJ}$. Table II summarizes conditions for $99.5 \%$ purity argon plasma at the lowest and highest energies we have operated the device, with typical conditions in the middle column. The lower limit of operation is set by the minimum voltage at which breakdown is possible.

In high-power configurations, a shock wave is generated by rapid Joule heating. The sudden and large overpressure can fracture the quartz tube and destroy the test samples. The upper limit of operation of $150 \mathrm{MW}$ is determined by mechanical failure of the chamber. A majority of the plasma/surface interaction studies were conducted at typical conditions. Typical conditions correspond to four capacitors arranged in a series-parallel configuration, charged to $3.2 \pm$ $0.1 \mathrm{kV}$ with argon flowing at $45 \pm 2$ SLPM.

Fig. 5 shows typical normalized voltage, current, and light intensity waveforms for breakdown in $99.5 \%$ pure argon using four capacitors at $3.2 \mathrm{kV}$. Voltage and current traces are underdamped at typical operating conditions, and the light intensity 
TABLE III

OBSERVEd Lines AND AsSOCiATEd ENERGy LEVELS For 99.5\% PURE ARgon Plasma AT TyPiCAL OPERATING CONDITIONS

\begin{tabular}{|c|c|c|c|c|c|}
\hline \multirow[t]{2}{*}{ Species } & \multirow[t]{2}{*}{ Wavelength } & \multicolumn{2}{|c|}{$\begin{array}{l}\text { Lower Energy } \\
\text { Levels }\end{array}$} & \multicolumn{2}{|c|}{ "Upper Energy levels } \\
\hline & & $\mathrm{E}_{\mathrm{i}}(\mathrm{eV})$ & $\mathrm{J}_{\mathrm{i}}$ & $\mathrm{E}_{\mathrm{k}}(\mathrm{eV})$ & $\mathrm{J}_{\mathrm{k}}$ \\
\hline \multirow{2}{*}{ Ar III } & 250.89 & 23.14 & 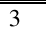 & 28.08 & 3 \\
\hline & 274.38 & 23.39 & 3 & 27.91 & 2 \\
\hline \multirow{7}{*}{ Ar II } & 384.54 & 16.64 & $5 / 2$ & 19.86 & $3 / 2$ \\
\hline & 434.80 & 16.64 & $5 / 2$ & 19.49 & $7 / 2$ \\
\hline & 440.09 & 16.40 & $7 / 2$ & 19.22 & $\begin{array}{l}5 / 2 \\
3 / 2\end{array}$ \\
\hline & 472.68 & 17.14 & $3 / 2$ & 19.76 & $5 / 2$ \\
\hline & 480.60 & 16.64 & $5 / 2$ & 19.22 & $5 / 2$ \\
\hline & 487.98 & 17.14 & $3 / 2$ & 19.68 & $\begin{array}{l}5 / 2 \\
5 / 2\end{array}$ \\
\hline & 501.71 & 18.65 & $3 / 2$ & 21.12 & $5 / 2$ \\
\hline Si II & 634.71 & 8.121 & $1 / 2$ & 10.07 & $3 / 2$ \\
\hline $\mathrm{H}_{\alpha}$ & 656.52 & 10.19 & $3 / 2$ & 12.08 & $5 / 2$ \\
\hline \multirow{17}{*}{ Ar I } & 696.02 & 11.54 & 2 & 13.32 & 1 \\
\hline & 706.72 & 11.54 & 2 & 13.30 & 2 \\
\hline & 714.70 & 11.54 & 2 & 13.28 & 1 \\
\hline & 727.29 & 11.62 & 1 & 13.32 & 1 \\
\hline & 738.39 & 11.62 & 1 & 13.30 & 2 \\
\hline & 750.38 & 11.82 & 1 & 13.47 & 0 \\
\hline & 763.51 & 11.54 & 2 & 13.17 & 2 \\
\hline & 772.37 & 11.54 & 2 & 13.15 & 1 \\
\hline & 772.42 & 11.72 & 0 & 13.32 & 1 \\
\hline & 794.81 & 11.72 & 0 & 13.28 & 1 \\
\hline & 800.61 & 11.62 & 1 & 13.17 & 2 \\
\hline & 810.36 & 11.62 & 1 & 13.15 & 1 \\
\hline & 826.45 & 11.82 & 1 & 13.32 & 1 \\
\hline & 840.82 & 11.82 & 1 & 13.30 & 2 \\
\hline & 842.46 & 11.62 & 1 & 13.09 & 2 \\
\hline & 852.14 & 11.81 & 1 & 11.62 & 2 \\
\hline & 912.29 & 11.54 & 2 & 12.90 & 1 \\
\hline
\end{tabular}

waveform lags the current. The light emission continues at a low level after the plasma current has reached zero.

\section{Emission Spectra of the Thermal Discharge}

Time integrated emission spectra of the discharge were recorded by the Ocean Optics spectrometer with a wavelength range from 200 to $1000 \mathrm{~nm}$. The plasma was viewed with an optical fiber and lens that looked either across the diameter of the plasma source $(L \sim 32 \mathrm{~mm})$ or along the length $(L \sim 100 \mathrm{~mm})$ of the chamber. The light emission from the plasma source was dominated by lines of neutral Ar I and ionized Ar II. In addition, emission lines from silicon and hydrogen $\left(H_{\alpha}\right)$ were also observed. Table III summarizes the observed lines and associated energy levels for the argon arc at typical operating conditions.

Plasma temperatures were estimated between 1 and $2 \mathrm{eV}$ assuming a Boltzmann equilibrium distribution for all excited species. The equilibrium Boltzmann assumption is valid for pressures of $\sim 10^{5} \mathrm{~Pa}$ and ionization ratios of $\sim 10^{-3}$ [12]. The spectrum of the discharge for typical operating conditions is shown in Fig. 6.

Some discharges showed line radiation from silicon neutral atoms in the spectral region $\lambda \sim 250 \mathrm{~nm}$. Silicon neutral lines in the spectrum indicate ablation of the quartz chamber walls.

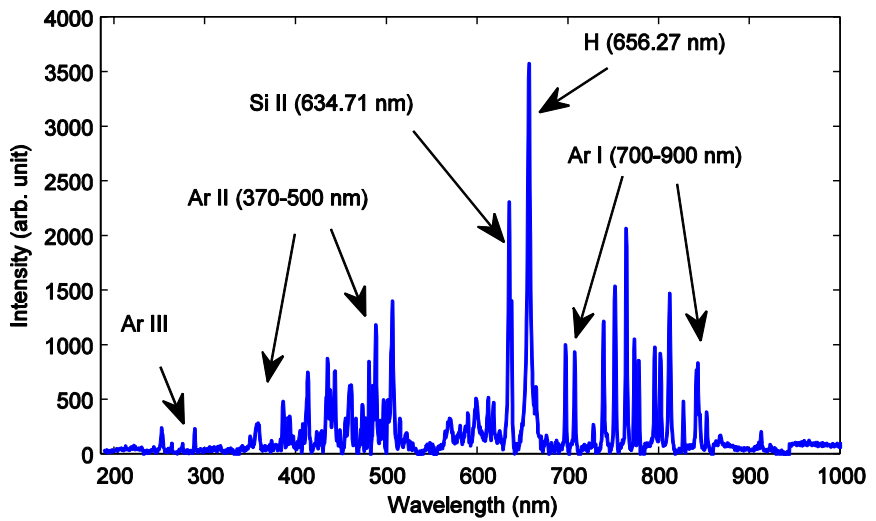

Fig. 6. Corrected emission spectrum in the wavelength range of $200-1000 \mathrm{~nm}$ for $\mathrm{Ar}+2 \% \mathrm{H}_{2}$ discharge at $4 \mathrm{kV}$. The observed dominant emission lines of $\mathrm{Ar}$ I, Ar II, Ar III, H, and Si II are seen in the spectrum. Electron temperature $\left(\mathrm{T}_{\mathrm{e}}\right)$ estimated from the intensity of emission line ratios using the Boltzmann plot method.

Surface analysis of the quartz chamber wall and test materials confirmed the presence of silicon from the plasma chamber. The ablation of silicon from the quartz wall is discussed in more detail in Section IV.

In all spectra, the radiated power was dominated by line radiation. Optical depth considerations were important when analyzing the plasma spectra because the device operates in a high-density and high-pressure regime. Stark broadening of argon ion lines was too small to be resolved with the Ocean Optics spectrometer. With the addition of a small amount of hydrogen, time-averaged measurements of electron number density from the Stark broadened hydrogen lines were estimated to be $n_{e} \sim 10^{23} \mathrm{~m}^{-3}$.

\section{Time Evolution of the Thermal Plasma Discharge}

High-speed imaging has provided additional insights into the plasma evolution. The most important findings are: 1) the plasma discharge is constricted and does not occupy the available chamber volume and 2) multiple arcs can form, with a high degree of randomness to their shape. Consequently, there is significant shot-to-shot variation in how the core of the plasma interacts with samples in the reaction chamber.

Fig. 7(a) and (b) shows high-speed image sequences for two separate shots of the arc discharge. Fig. 7(a) shows a time evolution of the plasma arc discharge. The arc initially exhibits multiple branched filaments from the anode (left) at $10 \mu \mathrm{s}$. The arc filaments attach and track along the surface of the alumina sample-holder to the cathode. At $35 \mu$ s the arc filaments have coalesced into a single diffuse arc discharge. Fig. 7(b) shows the time evolution of another constricted arc filament bridging the interelectrode gap at 10 and $20 \mu$ s. There is minimal interaction with the sample holder. Initially, the arc occupies a small localized volume. As the current and temperature rises, the arc volume grows while retaining the initial geometric configuration.

The images show the high degree of randomness in the initial formation and development of the arc filament. Once an arc is formed, the diameter increases until the arc occupies 


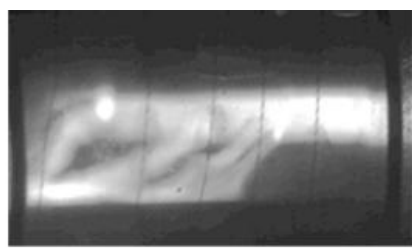

a)

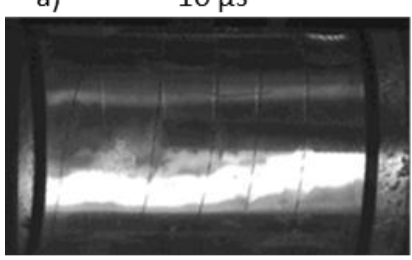

b)

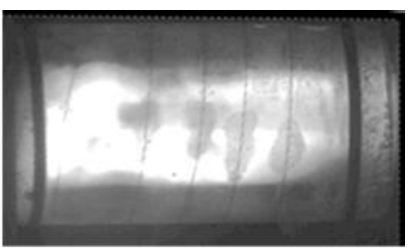

$35 \mu s$

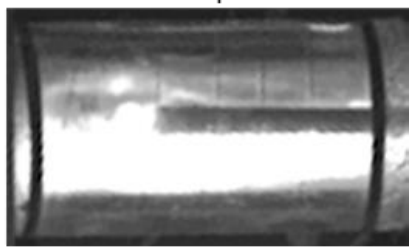

$20 \mu s$
Fig. 7. High-speed photography of the thermal arc discharge in argon. (a) Time evolution of filamentary branching and surface tracking along the sample holder at $10 \mu \mathrm{s}$, and growth of the filamentary arc at $35 \mu \mathrm{s}$. (b) Time evolution of the constricted plasma arc filament that does not interact with the sample holder at 10 and $20 \mu \mathrm{s}$.

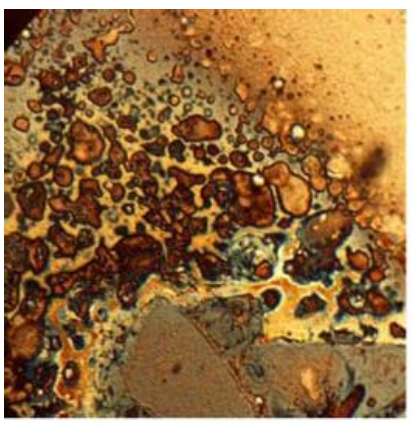

(a)

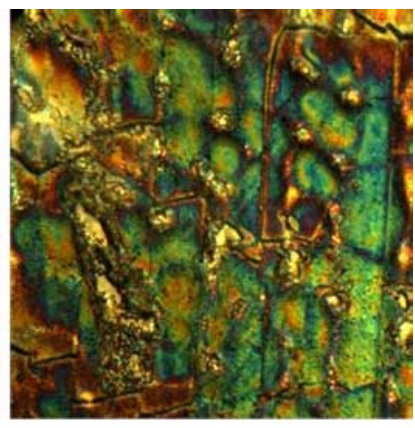

(b)
Fig. 8. 10× photomicrographs taken with a Nikon Optiphot 100 of (a) silicon and (b) sapphire showing deposits of copper from early tests using brass electrodes. Similar samples tested with Elkonite electrodes show no evidence of copper.

approximately a quarter of the chamber volume. Modifications to the design of the plasma chamber are likely required to create uniform and voluminous arcs that interact strongly with the samples. We are presently investigating smaller diameter reaction chambers where the quartz tube surface itself better defines and stabilizes the arc.

\section{Interaction of High-Density Plasma With SILICON AND SAPPHIRE SAMPLES}

Presently, the device has been used to study the interaction of thermal plasmas with silicon, alumina, and sapphire test samples orientated orthogonal to the flow. Initial studies were conducted using a brass anode and a steel cathode. In those studies, the samples became coated with copper from the anode, as shown in Fig. 8(a) and (b). To decrease electrode material deposition on test samples, electrodes were fabricated from Elkonite 50W3, an infiltrated 10\% copper and $90 \%$ tungsten contact material. The high-melting temperature of tungsten prevents evaporation of electrode material, when the arc is in contact with the surface. Copper possesses high

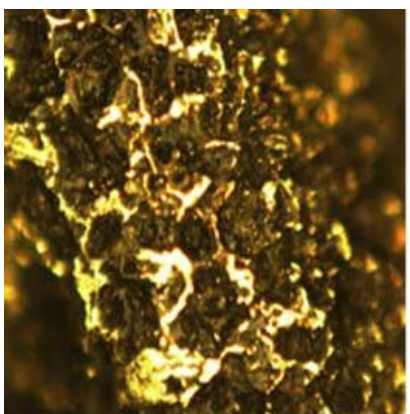

(a)

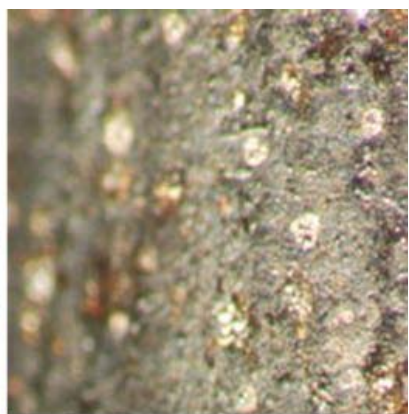

(b)
Fig. 9. 10× magnification photomicrographs of (a) brass and (b) Elkonite electrodes after several hundred tests. The brass shows gross removal of molten material.

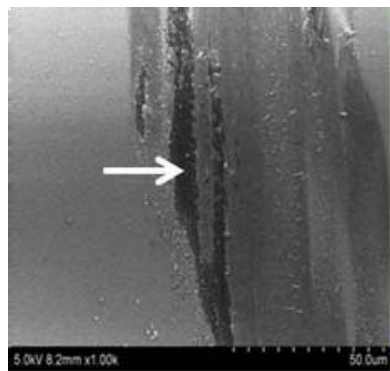

(a)

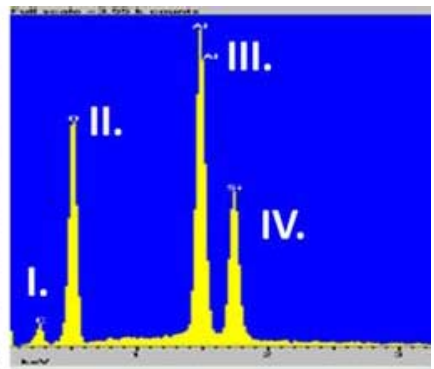

(b)
Fig. 10. (a) Surface of single-crystal sapphire showing an abundance of silicon deposited onto the sample. The arrow indicates where scratching of the surface exposed the sapphire substrate. (b) EDXS analysis of the sample confirming the presence of (I) carbon, (II) oxygen, (III) aluminum, and (IV) silicon.

thermal and electrical conductivity that allow large currents to flow and reduce local thermal loadings.

Before testing, the electrodes needed be conditioned. About 50 shots were required to evaporate the copper from the surface leaving only the tungsten. Fig. 9 compares the surface of the brass and Elkonite anodes. As expected, the brass shows evidence of gross melting from the arc, whereas the Elkonite shows only small spots that are presumably arc attachment spots on the Elkonite surface.

Evaporated silicon from the wall of the quartz chamber presented a second source of contamination. Silicon deposits were observed on the test samples. The quartz chamber and test samples were analyzed with a Hitachi S-4700 highresolution SEM equipped with an Oxford Instruments EDXS microanalysis system. Fig. 10(a) shows an SEM photograph of a single-crystal sapphire sample after 15 exposures to the plasma. The sample was coated with a fine dusting of silicon particles with an average particle size of $30-40 \mathrm{~nm}$. At the center of the photograph is an area that was gently touched with the edge of a set of fine tweezers, revealing the sapphire substrate that appears similar to the surface of unexposed sapphire. EDXS analysis of the sample, as shown in Fig. 10(b), confirms there was a significant amount of silicon on the surface of the sample as well as trace amounts of carbon contamination. 


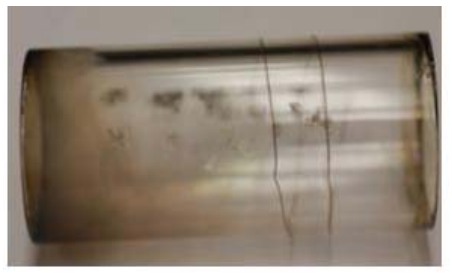

(a)

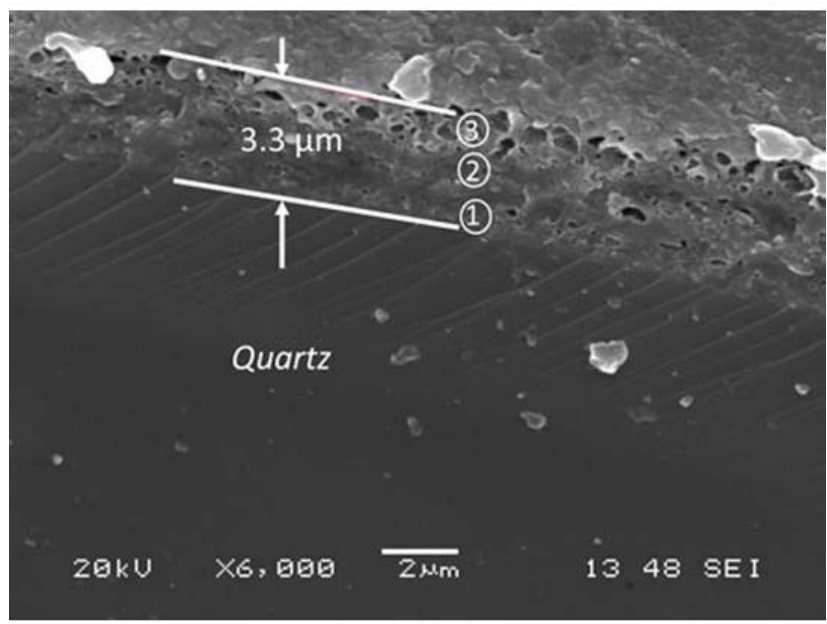

(c)

Fig. 11. (a) Plasma quartz chamber after almost 300 exposures. Left: the damaged part of the fused quartz was nearest to the anode, whereas the cathode end of the tube showed no damage (a small ring of discoloration on the cathode side is on the exterior of the tube, caused by the decomposition of an O-ring). (b) SEM analyses of the surface near the anode show an abundance of spheroidal holes. (c) SEM analysis of a chipped edge, also by the anode, shows that the depth of the affected zone is about $3.3 \mu \mathrm{m}$.

Fig. 11 shows a section of the quartz plasma chamber that was removed after approximately 300 exposures. The chamber was exposed to argon plasma at a charge voltage of $3.2 \mathrm{kV}$. The interior of the chamber closest to the gas inlet electrode (anode for this test sequence) was discolored and slightly opaque [left portion of quartz chamber Fig. 11(a)], whereas practically no damage was observed at the gas outlet [or cathode, right portion quartz chamber Fig. 11(a)]. SEM analysis of the sample from the damaged region showed the surface to contain a number of spheroidal pores on the order of $1 \mu \mathrm{m}$ in diameter, as shown in Fig. 11(b). Analysis of a cleaved cross section, as shown in Fig. 11(c), showed three distinct zones: number 1 is the boundary between the unaffected quartz and the reacted zone above, number 2 indicates a vitrification zone, and number 3 indicates a highly porous zone.

The damaged zone extends approximately 3.2-3.4 $\mu \mathrm{m}$ into the inner surface of the quartz chamber. Cross-sectional analysis from samples on the cathode side of the chamber did not show any damage. EDXS analysis of the quartz chamber anode region showed a significant silicon deficiency with respect to the control. Carbon contamination was also documented with EDXS that corresponds to the discoloration observed in Fig. 11(a). To prevent silicon deposition on test materials due to the accumulation of damage, the plasma chamber must be replaced after 100 exposures.
Several factors have been proposed to explain the asymmetrical erosion of the plasma chamber. These include polarity of the electrodes, orientation of the trigger wire, and proximity of the gas inlet electrode to the chamber wall. Reversing polarity of the electrodes does not change the erosion pattern; erosion still occurs at the gas inlet side of the reaction chamber. Similarly, reversing the orientation of the trigger wire did not change the location of the damage. Damage of the quartz chamber is likely due to the protrusion of the gas inlet electrode and the associated enhancement of the electric fields on this electrode.

\section{CONCLUSION}

A gas-fed plasma source has been developed to study how pulsed, high-density plasmas interact with materials. Plasma parameters for the pulsed atmospheric thermal discharge were determined, including the estimated electron temperature using the Boltzmann plot method, $T_{e} \sim 1-2 \mathrm{eV}$, and the estimated electron number density, $n_{e} \sim 10^{17} \mathrm{~cm}^{-3}$. The electron temperature and density are within the expected regime for high-density LTE discharges.

The use of feed gases to form the plasma arc provides a higher degree of control over the plasma composition than can be achieved using consumable liners and exploding fuse wire systems. Although a high degree of control of the plasma chemistry and minimization of contaminant materials has been achieved in the gas-fed plasma chamber, electrode, and chamber wall deterioration was observed. Contamination from the electrodes was minimized using Elkonite 50W3 as the electrode material. Elimination of the quartz chamber wall erosion is difficult due to high-thermal loads and localized streamer surface tracking. High-speed images of streamers and arcs show that the damage to the walls correlates strongly with the location of streamers between the gas inlet electrode and trigger wire.

High-speed imaging has also shown a high degree of variability in the arc formation. Arcs typically evolve to occupy about a quarter of the reaction chamber volume. Consequently, there is a large shot-to-shot variation in how the plasma core interacts with samples. The random shape of the arc column is a consequence of the random nature of the streamers and the large chamber volume. Although the arc structure is random, the current, light intensity, pressure, and emission spectra are highly reproducible.

The gas-fed plasma source that has been developed provides a controllable environment for material interaction investigations and the ability to reliably breakdown at pressuredistance products exceeding the Paschen breakdown threshold. The novel triggering approach provides reliable ignition that does not require the use of ablative liners or exploding wire ignition techniques that would contaminate the system as well as reduce throughput. The plasma device is ideal for highly controllable, low contamination, and plasma-material interaction studies.

\section{ACKNOWLEDGMENT}

The authors would like to thank S. Rojani and E. Trevino for their help. 


\section{REFERENCES}

[1] E. Stuhlinger, "Electric space propulsion systems," Space Sci. Rev., vol. 7, nos. 5-6, pp. 795-847, Dec. 1967.

[2] L. L. Raja, P. L. Varghese, and D. E. Wilson, "Modeling of the electrogun metal vapor plasma discharge," J. Thermophys. Heat Transf., vol. 11 , no. 3, pp. 353-360, Jul./Sep. 1997.

[3] P. J. Wilbur, R. G. Jahn, and F. C. Curran, "Space electric propulsion plasmas," IEEE Trans. Plasma Sci., vol. 19, no. 6, pp. 1167-1179, Dec. 1991.

[4] A. Gleizes, J. J. Gonzalez, and P. Freton, "Thermal plasma modelling," J. Phys. D, Appl. Phys., vol. 38, no. 9, pp. R153-R183, Apr. 2005

[5] E. Ahedo, "Plasmas for space propulsion," Plasma Phys. Controlled Fusion, vol. 53, no. 12, pp. 124037-124054, Nov. 2011.

[6] M. I. Boulos, "Thermal plasma processing," IEEE Trans. Plasma Sci., vol. 19, no. 6, pp. 1078-1089, Dec. 1991.

[7] L. Pekker and O. Pekker, "Model of high-pressure ablative capillary discharge for plasma thrusters," J. Propuls. Power, vol. 27, no. 2, pp. 477-484, Mar./Apr. 2011.

[8] C. B. Ruchti and L. Niemeyer, "Ablation controlled arcs," IEEE Trans. Plasma Sci., vol. 14, no. 4, pp. 423-434, Aug. 1986.
[9] D. E. Wilson, K. Kim, and L. L. Raja, "Theoretical analysis of an external pulsed plasma jet," IEEE Trans. Magn., vol. 35, no. 1 , pp. 228-233, Jan. 1999.

[10] J. D. Powell and A. E. Zielinski, "Capillary discharge in the electrothermal gun," IEEE Trans. Magn., vol. 29, no. 1, pp. 591-596, Jan. 1993.

[11] W. R. Hook, R. H. Dishington, and R. P. Hilberg, "Xenon flashlamp triggering for laser applications," IEEE Trans. Electron Devices, vol. 19, no. 3, pp. 308-314, Mar. 1972

[12] X.-M. Zhu and Y.-K. Pu, "Optical emission spectroscopy in low-temperature plasmas containing argon and nitrogen: Determination of the electron temperature and density by the line-ratio method," J. Phys. D, Appl. Phys., vol. 43, no. 40, p. 403001, Sep. 2010.

Authors' photographs and biographies not available at the time of publication. 